\title{
Fermion Scattering off a CP-violating Bubble Wall in the Background of a Uniform Magnetic Field
}

\author{
L. Campanelli ${ }^{1,2 *}$, G. L. Fogli ${ }^{3,4 \dagger}$ and L. Tedesco ${ }^{3,4 \ddagger}$ \\ ${ }^{1}$ Dipartimento di Fisica, Università di Ferrara, I-44100 Ferrara, Italy \\ ${ }^{2}$ INFN - Sezione di Ferrara, I-44100 Ferrara, Italy \\ ${ }^{3}$ Dipartimento di Fisica, Università di Bari, I-70126 Bari, Italy \\ ${ }^{4}$ INFN - Sezione di Bari, I-70126 Bari, Italy
}

September, 2004

\begin{abstract}
In the scenario of the electroweak baryogenesis we consider the dynamics of fermions with a spatially varying mass in presence of a CP-violating bubble wall and a uniform magnetic field perpendicular to the wall. The relevant quantity for baryogenesis, $R_{R \rightarrow L}-R_{L \rightarrow R}$, is studied $\left(R_{R \rightarrow L}\right.$ and $R_{L \rightarrow R}$ being the reflection coefficients for right-handed and left-handed chiral fermions, respectively).
\end{abstract}

PACS number(s): 12.15.Ji, 11.30.Fs, 98.80.Cq

\footnotetext{
${ }^{*}$ Electronic address: campanelli@fe.infn.it

$\dagger$ Electronic address: Fogli@ba.infn.it

${ }^{\ddagger}$ Electronic address: luigi.tedesco@ba.infn. it
} 


\section{Introduction}

The connection between particle physics and cosmology has received much attention in the last years. In fact, cosmology can use the predictions of particle physics in order to solve cosmological problems. The origin of the asymmetry between matter-antimatter is one of those important open questions in cosmology. As Sakharov pointed out more than thirty years ago [1, it is possible to start with a Universe in a baryon symmetry state and later dynamically to generate a net asymmetry by particle interactions. He postulated three conditions in order to explain the observed baryon excess: 1) Baryon number non-conservation; this violation occurs in the Standard Model [2]-4] through the axial anomaly, but the rate of baryon number non-conserving processes are exponentially suppressed at zero temperature. 2) $C$ and $C P$-violation; this violation has been observed in the neutral $\mathrm{K}$ meson system and recently in the B meson decay [5]. 3) Departure from thermal equilibrium; in fact, in thermal equilibrium particles and antiparticles will have the same number density.

The Standard Model has in principle all the necessary ingredients for Sakharov conditions for the generation of the baryon asymmetry of the Universe [4. In particular the electroweak baryogenesis is a good candidate because give us an explanation in terms of experimentally accessible physics. Essentially the baryon asymmetry problem is twofold to understand: 1) why the observable density of baryons in the Universe is much greater than the density of the antibaryons and 2) why the density of baryons is much less than density of photons. Indeed, the measurement of the ratio of baryon density $n_{B}$ (defined as the number density of baryons $n_{b}$ minus the number density of antibaryons $n_{\bar{b}}$ ) and the photon density $n_{\gamma}$, at the present time, recently has been determined from the measurement of the relative heights of the first two CMB acoustic peaks by WMAP satellite. It takes the value [6]

$$
5.9 \times 10^{-10} \leq \frac{n_{B}}{n_{\gamma}} \leq 6.4 \times 10^{-10} .
$$

$\mathrm{CP}$-violation is crucial to generate the baryon asymmetry. It is well known, however, that the amount of CP-violation in the quark sector of the standard model cannot account for the observed baryon asymmetry [7, 8]. In fact, the strength of CP-violation coming from the CKM phase is inadequate by 10-12 orders of magnitude $\left(n_{B} / n_{\gamma} \sim 10^{-20}\right)[9$.

Another question is connected with the third Sakharov condition. The baryogenesis necessarily requires non-equilibrium physics. In order to avoid the so called washout after the phase transition, it is necessary to have $v\left(T_{c}\right) / T_{c} \geq 1$, where $v\left(T_{c}\right)$ is the vacuum expectation value of the broken Higgs field. This condition ensures the surviving of the baryon asymmetry after the walls has passed and indicates that the electroweak phase transition should be, if strong enough, of the first order [10. Moreover, lattice studies have shown that for any Higgs mass, the phase transition would be so weak that sphaleron interactions are in equilibrium in the broken phase. This implies that the baryon asymmetry goes to zero immediately after its generation [11. On the other hand, if we use one loop high temperature effective potential we have an Higgs boson mass $M_{H} \leq 60 \mathrm{Gev}$ [12] which is in contrast with the experimental lower bound reached $M_{H}>114,4 \mathrm{Gev}$ [13]. Hence, the magnitude of CP-violation and the experimental limits on Higgs mass contradict the possibility to have an asymmetry of the experimentally observed order within the framework of the Minimal Standard Model and one is lead to consider extensions of the Standard Model. If we demands that the electroweak phase transition has to be of first order, we 
have a mechanism for the transition called bubble nucleation. It proceeds by formation and expansion of bubbles of new phase within the old ones. The study of the propagation of the bubble has been object of much attention as from the early works [14], 15]. The first order provided a natural way to depart from equilibrium that takes places near the wall of the expanding bubbles. Moreover, in the unbroken phase where the sphaleron is active, the asymmetries of some local charges are converted into a baryon asymmetry. The baryon number flows into the new phase and the baryogenesis is produced. In this scenario of electroweak baryogenesis there are two interesting mechanisms that involve interacting fermions with CP-violating background of the bubble. In both cases, it is possible to have a net baryon flux by using fermion scattering off bubble walls [16]: The local baryogenesis [17], [18] in which the CP-violating and B-violating processes are near or at the bubble wall. 2) Non local baryogenesis [16] (or charge transport mechanism) in which CP-violation and baryon number violation are separated from one another.

In our work we consider this last kind of electroweak baryogenesis. Moreover, the non local baryogenesis is effective for thin-wall regime [16]-[19]. Hence, it is possible to treat as free the fermions that may only interact in a small region with Higgs background field. As a consequence of CP-violation on the wall, we will have asymmetric reflection and transmission, and the particles injected into the broken phase diffuse in the bubble. The correct way to treat the generation of the asymmetry in front of the bubble is controversial. A very simple mode is to consider an extra source of CP-violation, parameterized assigning a spatially varying complex mass to the Higgs field:

$$
m(t, \vec{x})=m_{R}(t, \vec{x})+i m_{I}(t, \vec{x}) .
$$

The imaginary part $m_{I}$ gives rise to CP-violation. The problem to solve Dirac equation in the background of the bubble wall without CP-violation [20] and with CP-violation [21] has been already studied. The authors also have studied the case of fermion scattering in the background of the bubble wall without CP-violation and in presence of a constant magnetic field perpendicular to the wall [22].

It is important to stress that the introduction of a magnetic field is not an academic exercise because astronomical observations show the presence of a cosmic magnetic field in all galaxies [23]. There are some possible mechanism for generating a cosmological magnetic field in the early Universe. One of these is related to the first order electroweak phase transition [24], in which, bubbles of the new phase expanding in the old ones generate electric currents that, in turns, produce magnetic fields [25].

In this paper we study and solve the Dirac equation in presence of a CP-violating planar bubble wall with a constant magnetic field perpendicular to the wall. We consider the scattering perpendicular to the wall and the radius of the bubble very large. Neglecting the time dependence of mass terms, we shall work in the hypothesis that $m(t, \vec{x})$ only depends by the $z$-coordinate perpendicular to the wall: $m(t, \vec{x})=m(z)$. The real part goes to zero when $z \rightarrow-\infty$ (symmetric phase) and goes to $m_{0}$ (broken phase) when $z \rightarrow+\infty$, where $m_{0}$ is fermion mass. We calculate the reflection coefficients, $R_{R \rightarrow L}$ and $R_{L \rightarrow R}$, of lefthanded and right-handed fermions, respectively, at a radially expanding bubble. The difference $\Delta R=R_{R \rightarrow L}-R_{L \rightarrow R}$ is important as regards the baryon asymmetry [26] in cosmology. 


\section{CP-violating Dirac equation}

In this Section we study the scattering of Dirac fermions off CP-violating bubble walls in the presence of an electromagnetic field $A_{\mu}$. Thus, assuming that a fermion $\Psi$ is coupled to a complex scalar field $\Phi$ through a Yukawa interaction with coupling $g_{Y}$, the Dirac equation reads:

$$
\left[i \not \partial-m(t, \vec{x}) P_{R}-m^{*}(t, \vec{x}) P_{L}-e \not A\right] \Psi(t, \vec{x})=0,
$$

where $e$ is the electric charge, $P_{R}=\left(1+\gamma_{5}\right) / 2$ and $P_{L}=\left(1-\gamma_{5}\right) / 2$, are the right-handed and left-handed projection operators such that $P_{R} P_{L}=P_{L} P_{R}=0, P_{R}^{2}=P_{R}, P_{L}^{2}=P_{L}$, and

$$
m(t, \vec{x})=-g_{Y}\langle\Phi(t, \vec{x})\rangle
$$

is a complex function of space-time. We only consider the motion of fermions perpendicular to the wall, and the radius of the bubble very large in order to approximate the surface by a flat wall perpendicular to the $z$-axis. Hence, we shall consider $m(t, \vec{x})=m(z)$. In order to solve Eq. (2.1), we make the following ansatz:

$$
\Psi(t, z)=\left[i \not \partial+m(z) P_{R}+m^{*}(z) P_{L}-e \not A\right] e^{-i \sigma E t} \psi_{E}(z)
$$

where $\sigma= \pm 1$ for positive and negative energy solutions, respectively. Moreover, we assume that $A_{\mu}$ corresponds to a constant and uniform magnetic field directed along the $z$-axis with strength $B$. We can then choose the Landau gauge

$$
A_{\mu}=(0,0,-B x, 0) .
$$

Inserting Eq. (2.3) into Eq. (2.1), we get

$$
\left[E^{2}+\frac{d^{2}}{d z^{2}}-|m|^{2}+i \gamma^{3} \frac{d m_{R}}{d z}+\gamma^{3} \gamma_{5} \frac{d m_{I}}{d z}+i e B \gamma^{1} \gamma^{2}\right] \psi_{E}(z)=0
$$

It is useful to introduce a parameter mass term $a,(1 / a$ being the characteristic size of the thickness of the wall) and define

$$
\begin{aligned}
m_{R}(z) & =m_{0} f(a z)=m_{0} f(x), \\
m_{I}(z) & =m_{0} g(a z)=m_{0} g(x),
\end{aligned}
$$

where

$$
x=a z, \quad \tau=a t, \quad \epsilon=E / a, \quad \xi=m_{0} / a, \quad b=e B / a^{2} .
$$

Taking into account Eqs. (2.6)-(2.8), the ansatz (2.3) and Eq. (2.5) become, respectively

$$
\begin{aligned}
& \Psi(\tau, x)=\left[\sigma \epsilon \gamma^{0}+i \gamma^{3} \frac{d}{d x}+\xi f-i \xi g \gamma_{5}+b x \gamma^{2}\right] e^{-i \sigma \epsilon \tau} \psi_{\epsilon}(x), \\
& {\left[\epsilon^{2}+\frac{d^{2}}{d x^{2}}-\xi^{2}\left(f^{2}+g^{2}\right)+i \xi \gamma^{3} f^{\prime}-\xi \gamma_{5} \gamma^{3} g^{\prime}+i b \gamma^{1} \gamma^{2}\right] \psi_{\epsilon}(x)=0}
\end{aligned}
$$

where a prime denotes differentiation with respect to $x$. Let us suppose that $|g(x)| \ll 1$. The small value of $g$ allows to consider it as a perturbation and to consider only first order terms in $g$. We assume that

$$
\lim _{x \rightarrow+\infty} f(x)=1, \quad \lim _{x \rightarrow-\infty} f(x)=0 .
$$


In first order approximation we write

$$
\psi_{\epsilon}(x)=\psi^{(0)}(x)+\psi^{(1)}(x) .
$$

The wave function $\psi^{(0)}$ is a solution of the unperturbed equation

$$
\left[\epsilon^{2}+\frac{d^{2}}{d x^{2}}-\xi^{2} f^{2}+i \xi \gamma^{3} f^{\prime}+i b \gamma^{1} \gamma^{2}\right] \psi^{(0)}(x)=0,
$$

which is obtained from Eq. (2.10) with $g=0$. The perturbation $\psi^{(1)}$ can be calculated as

$$
\psi^{(1)}(x)=\int d x^{\prime} G\left(x, x^{\prime}\right) V\left(x^{\prime}\right) \psi^{(0)}\left(x^{\prime}\right),
$$

where $V(x)=-\xi g\left(x^{\prime}\right) \gamma_{5} \gamma^{3}$, and $G\left(x, x^{\prime}\right)$ is a Green function that satisfies the following equation,

$$
\left[\epsilon^{2}+\frac{d^{2}}{d x^{2}}-\xi^{2} f^{2}+i \xi f^{\prime} \gamma^{3}+i b \gamma^{1} \gamma^{2}\right]_{\alpha \beta} G_{\beta \gamma}\left(x, x^{\prime}\right)=-\delta_{\alpha \gamma} \delta\left(x-x^{\prime}\right) .
$$

Inserting Eq. (2.12) in Eq. (2.10) we have

$$
\Psi(\tau, x) \simeq\left[\left(\sigma \epsilon \gamma^{0}+i \gamma^{3} \frac{d}{d x}+\xi f+b x \gamma^{2}\right)\left(\psi^{(0)}+\psi^{(1)}\right)+i \xi g \gamma_{5} \psi^{(0)}\right] e^{-i \sigma \epsilon \tau},
$$

to the first order in $g$. In order to obtain $\psi^{(0)}$ we have to solve Eq. (2.13) (see Refs. [21], [22]). Let us expand $\psi^{(0)}$ in terms of the eigenstates of $\gamma^{3}$ :

$$
\psi^{(0)}(x)=\phi_{ \pm}^{(s)}(x) u_{ \pm}^{s}
$$

where $s=1,2$, and

$$
u_{ \pm}^{1}=\frac{1}{\sqrt{2}}\left(\begin{array}{c}
1 \\
0 \\
\pm i \\
0
\end{array}\right), \quad u_{ \pm}^{2}=\frac{1}{\sqrt{2}}\left(\begin{array}{c}
0 \\
1 \\
0 \\
\mp i
\end{array}\right)
$$

The spinors $u_{ \pm}^{s}$ satisfy the relations

$$
\begin{aligned}
\gamma^{0} u_{ \pm}^{s} & =u_{\mp}^{s}, \\
\gamma^{1} \gamma^{2} u_{ \pm}^{s} & =i(-1)^{s} u_{ \pm}^{s}, \\
\gamma^{3} u_{ \pm}^{s} & = \pm i u_{ \pm}^{s}, \\
\gamma_{5} u_{ \pm}^{s} & =\mp i(-1)^{s} u_{\mp}^{s},
\end{aligned}
$$

that will be useful in the following. Inserting Eq. (2.17) into Eq. (2.13), we get

$$
\left[\epsilon^{2}+\frac{d^{2}}{d x^{2}}-\xi^{2} f^{2} \mp \xi f^{\prime}-(-1)^{s} b\right] \phi_{ \pm}^{(s)}(x)=0 .
$$


We indicate the independent solutions of Eq. (2.23) as $\phi_{ \pm}^{\left(+\alpha_{s}\right)}(x)$ and $\phi_{ \pm}^{\left(-\alpha_{s}\right)}(x)$. The asymptotic properties of $\phi_{ \pm}^{\left(+\alpha_{s}\right)}$ and $\phi_{ \pm}^{\left(-\alpha_{s}\right)}$ are, respectively

$$
\begin{aligned}
& \phi_{ \pm}^{\left(+\alpha_{s}\right)}(x) \rightarrow \begin{cases}e^{+\alpha_{s} x} & \text { for } x \rightarrow+\infty \\
\gamma_{ \pm}\left(\alpha_{s}, \beta_{s}\right) e^{\beta_{s} x}+\gamma_{ \pm}\left(\alpha_{s},-\beta_{s}\right) e^{-\beta_{s} x} & \text { for } x \rightarrow-\infty\end{cases} \\
& \phi_{ \pm}^{\left(-\alpha_{s}\right)}(x) \rightarrow \begin{cases}e^{-\alpha_{s} x} & \text { for } x \rightarrow+\infty \\
\gamma_{ \pm}\left(-\alpha_{s}, \beta_{s}\right) e^{\beta_{s} x}+\gamma_{ \pm}\left(-\alpha_{s},-\beta_{s}\right) e^{-\beta_{s} x} & \text { for } x \rightarrow-\infty\end{cases}
\end{aligned}
$$

where

$$
\begin{aligned}
& \alpha_{s}=i \sqrt{\epsilon^{2}-\xi^{2}-(-1)^{s} b}, \\
& \beta_{s}=i \sqrt{\epsilon^{2}-(-1)^{s} b},
\end{aligned}
$$

and $\gamma_{ \pm}\left(\alpha_{s}, \beta_{s}\right)$ are constants such that $\gamma_{ \pm}\left(\alpha_{s}, \beta_{s}\right)^{*}=\gamma_{ \pm}\left(-\alpha_{s},-\beta_{s}\right)$. The general unperturbed solution is

$$
\psi^{(0)}(x)=\sum_{s=1,2}\left[A_{+}^{\left(-\alpha_{s}\right)} \phi_{+}^{\left(-\alpha_{s}\right)}(x)+A_{+}^{\left(+\alpha_{s}\right)} \phi_{+}^{\left(+\alpha_{s}\right)}(x)\right] .
$$

The incident wave function coming from $x=-\infty$ is reflected and transmitted, while at $x=+\infty$ there is only the transmitted wave. Thus, we have $A^{\left(-\alpha_{s}\right)}=0$ for $\sigma=+1$ and $A^{\left(+\alpha_{s}\right)}=0$ for $\sigma=-1$.

Following Ref. 21, in order to calculate the Green function $G\left(x, x^{\prime}\right)$, we introduce a unitary matrix

$$
U=\left(u_{+}^{1} u_{-}^{1} u_{+}^{2} u_{-}^{2}\right)=\frac{1}{\sqrt{2}}\left(\begin{array}{cccc}
1 & 1 & 0 & 0 \\
0 & 0 & 1 & 1 \\
i & -i & 0 & 0 \\
0 & 0 & -i & i
\end{array}\right)
$$

and we write $G\left(x, x^{\prime}\right)$ as

$$
G\left(x, x^{\prime}\right)=U\left(\begin{array}{cccc}
G_{+}\left(x, x^{\prime}\right) & & & \\
& G_{-}\left(x, x^{\prime}\right) & & \\
& & G_{+}\left(x, x^{\prime}\right) & \\
& & G_{-}\left(x, x^{\prime}\right)
\end{array}\right) U^{-1}
$$

where

$$
\left[\frac{d^{2}}{d x^{2}} \mp \xi f^{\prime}-\xi^{2} f^{2}+\epsilon^{2}-(-1)^{s} b\right] G_{ \pm}\left(x, x^{\prime}\right)=-\delta\left(x-x^{\prime}\right) .
$$

Following the standard method [27, we find for the Green function the expression

$$
G_{ \pm}^{(s, \sigma)}\left(x, x^{\prime}\right)= \begin{cases}-\frac{\sigma}{2 \alpha_{s}}\left[\phi_{ \pm}^{\left(-\sigma, \alpha_{s}\right)}(x)+c_{ \pm}^{(s, \sigma)} \phi_{ \pm}^{\left(+\sigma, \alpha_{s}\right)}(x)\right] \phi_{ \pm}^{\left(-\sigma, \alpha_{s}\right)}\left(x^{\prime}\right) & \text { if } x<x^{\prime} \\ -\frac{\sigma}{2 \alpha_{s}}\left[\phi_{ \pm}^{\left(-\sigma, \alpha_{s}\right)}\left(x^{\prime}\right)+c_{ \pm}^{(s, \sigma)} \phi_{ \pm}^{\left(+\sigma, \alpha_{s}\right)}\left(x^{\prime}\right)\right] \phi_{ \pm}^{\left(-\sigma, \alpha_{s}\right)}(x) & \text { if } x>x^{\prime}\end{cases}
$$


Therefore, if we know the Green function and wave function $\psi^{(0)}$ it is possible to find, by Eq. (2.14), the wave function $\psi^{(1)}$ :

$$
\begin{aligned}
\psi_{s}^{(1)}(x) & =A_{s}^{(+)} \frac{(-1)^{s} \xi}{2 \alpha_{s}} u_{-}^{(s)} \\
& \times\left\{\phi_{-}^{\left(+\alpha_{s}\right)}(x) \int_{-\infty}^{x} d x^{\prime} g^{\prime}\left(x^{\prime}\right)\left[\phi_{-}^{\left(-\alpha_{s}\right)}\left(x^{\prime}\right)+c_{-}^{(+)} \phi_{-}^{\left(+\alpha_{s}\right)}\left(x^{\prime}\right)\right] \phi_{+}^{\left(+\alpha_{s}\right)}\left(x^{\prime}\right)\right. \\
& \left.+\left[\phi_{-}^{\left(-\alpha_{s}\right)}(x)+c_{-}^{(+)} \phi_{-}^{\left(+\alpha_{s}\right)}(x)\right] \int_{x}^{\infty} d x^{\prime} g^{\prime}\left(x^{\prime}\right) \phi_{-}^{\left(+\alpha_{s}\right)}\left(x^{\prime}\right) \phi_{+}^{\left(+\alpha_{s}\right)}\left(x^{\prime}\right)\right\} .
\end{aligned}
$$

Let us introduce the following quantities:

$$
\begin{aligned}
I_{1}^{(s)} & =\int_{-\infty}^{+\infty} d x g^{\prime}(x) \phi_{-}^{\left(-\alpha_{s}\right)}(x) \phi_{+}^{\left(+\alpha_{s}\right)}(x) \\
I_{2}^{(s)} & =\int_{-\infty}^{+\infty} d x g^{\prime}(x) \phi_{-}^{\left(+\alpha_{s}\right)}(x) \phi_{+}^{\left(+\alpha_{s}\right)}(x) .
\end{aligned}
$$

In Appendix A we shall calculate the asymptotic expression of the transmitted, incident and reflected wave functions (for definiteness, let us consider the case $\sigma=+1$ ). They are respectively,

$$
\begin{aligned}
{\left[\Psi_{s}(\tau, x)\right]_{\sigma=+1}^{\mathrm{tran}} } & =A_{s}^{(+)} e^{-i \epsilon \tau+\alpha_{s} x} \\
& \times\left\{\left(\xi-\alpha_{s}\right)\left[1+\frac{(-1)^{s} \xi\left(\xi+\alpha_{s}\right) I_{1}^{(s)}}{2 \epsilon \alpha_{s}}+\frac{\xi b\left(I_{1}^{(s)}+c_{-}^{(+)} I_{2}^{(s)}\right)}{2 \epsilon \alpha_{s}\left(\xi-\alpha_{s}\right)}+\frac{(-1)^{s} \xi g_{-}}{2 \epsilon}\right] u_{+}^{s}\right. \\
& \left.+\epsilon\left[1+\frac{(-1)^{s} \xi\left(\xi+\alpha_{s}\right) I_{1}^{(s)}}{2 \epsilon \alpha_{s}}-\frac{(-1)^{s} \xi g_{+}}{\epsilon}+\frac{(-1)^{s} \xi g_{-}}{2 \epsilon}\right] u_{-}^{s}\right\}, \\
{\left[\Psi_{s}(\tau, x)\right]_{\sigma=+1}^{\mathrm{inc}} } & =A_{s}^{(+)} \gamma_{+}\left(\alpha_{s}, \beta_{s}\right) e^{-i \epsilon \tau+\beta_{s} x} \\
& \times\left\{-\beta_{s}\left[1-\frac{(-1)^{s} \xi \epsilon I_{2}^{(s)}}{2 \alpha_{s} \beta_{s}} \frac{\gamma_{-}\left(-\alpha_{s}, \beta_{s}\right)}{\gamma_{+}\left(\alpha_{s}, \beta_{s}\right)}+\frac{\xi b c_{-}^{(+)} I_{2}^{(s)}}{2 \epsilon \alpha_{s}\left(\xi-\alpha_{s}\right)}+\frac{(-1)^{s} \xi g_{-}}{2 \epsilon}\right] u_{+}^{s}\right. \\
& \left.+\epsilon\left[1+\frac{(-1)^{s} \xi \beta_{s} I_{2}^{(s)}}{2 \epsilon \alpha_{s}} \frac{\gamma_{-}\left(-\alpha_{s}, \beta_{s}\right)}{\gamma_{+}\left(\alpha_{s}, \beta_{s}\right)}-\frac{(-1)^{s} \xi g_{-}}{2 \epsilon}\right] u_{-}^{s}\right\}, \\
{\left[\Psi_{s}(\tau, x)\right]_{\sigma=+1}^{\mathrm{refl}} } & =\left.\left[\Psi_{s}(\tau, x)\right]_{\sigma=+1}^{\mathrm{inc}}\right|_{\beta_{s} \rightarrow-\beta_{s}},
\end{aligned}
$$

where $g_{ \pm}=\lim _{x \rightarrow \pm \infty} g(x)$. From Eqs. (2.36)-(2.38) we calculate the vectorial current $j_{V}^{\mu}=\bar{\Psi} \gamma^{\mu} \Psi$ and the axial current $j_{A}^{\mu}=\bar{\Psi} \gamma^{\mu} \gamma_{5} \Psi$. After some manipulations we obtain

$$
\begin{aligned}
\left(j_{V, s}^{3}\right)^{\text {tran }} & =2 \epsilon\left|A_{s}^{(+)}\right|^{2}\left|\alpha_{s}\right|\left(1+\delta^{\text {tran }}\right) \\
\left(j_{V, s}^{3}\right)^{\text {inc }} & =2 \epsilon\left|A_{s}^{(+)}\right|^{2}\left|\gamma_{+}\left(\alpha_{s}, \beta_{s}\right)\right|^{2}\left|\beta_{s}\right|\left(1+\delta_{1}^{\text {inc }}+\delta_{2}^{\text {inc }}\right), \\
\left(j_{V, s}^{3}\right)^{\text {refl }} & =-\left.\left(j_{V, s}^{3}\right)^{\text {inc }}\right|_{\beta_{s} \rightarrow-\beta_{s}},
\end{aligned}
$$


where

$$
\begin{aligned}
\delta^{\operatorname{tran}} & =\frac{\xi b}{2 \epsilon|\alpha|_{s}^{2}} \operatorname{Re}\left[I_{1}^{(s)}+c_{-}^{(+)} I_{2}^{(s)}\right], \\
\delta_{1}^{\text {inc }} & =(-1)^{s} \frac{\xi\left|\beta_{s}\right|}{\epsilon\left|\alpha_{s}\right|} \operatorname{Re}\left[\frac{\gamma_{-}\left(-\alpha_{s}, \beta_{s}\right)}{\gamma_{+}\left(\alpha_{s}, \beta_{s}\right)} I_{2}^{(s)}\right], \\
\delta_{2}^{\text {inc }} & =\frac{\xi b}{2 \epsilon\left|\alpha_{s}\right|\left|\beta_{s}\right|}\left\{\operatorname{Re}\left[\frac{\gamma_{-}\left(-\alpha_{s}, \beta_{s}\right)}{\gamma_{+}\left(\alpha_{s}, \beta_{s}\right)} I_{2}^{(s)}\right]+\operatorname{Im}\left[\frac{i \beta_{s} c_{-}^{(+)}}{\alpha_{s}-\xi} I_{2}^{(s)}\right]\right\} .
\end{aligned}
$$

The transmission and reflection coefficients are

$$
\begin{aligned}
& T_{s, B}=\frac{\left(j_{V, s}^{3}\right)^{\text {tran }}}{\left(j_{V, s}^{3}\right)^{\text {inc }}}=T_{s, B}^{(0)}\left(1+\delta^{\text {tran }}-\delta_{1}^{\text {inc }}-\delta_{2}^{\text {inc }}\right) \\
& R_{s, B}=-\frac{\left(j_{V, s}^{3}\right)^{\text {refl }}}{\left(j_{V, s}^{3}\right)^{\text {inc }}}=R_{s, B}^{(0)}\left(1+\delta_{1}^{\text {refl }}+\delta_{2}^{\text {refl }}-\delta_{1}^{\text {inc }}-\delta_{2}^{\text {inc }}\right)
\end{aligned}
$$

where

$$
T_{s, B}^{(0)}=\frac{\left|\alpha_{s}\right|}{\left|\beta_{s}\right|\left|\gamma_{+}\left(\alpha_{s}, \beta_{s}\right)\right|^{2}}, \quad R_{s, B}^{(0)}=\left|\frac{\gamma_{+}\left(\alpha_{s},-\beta_{s}\right)}{\gamma_{+}\left(\alpha_{s}, \beta_{s}\right)}\right|^{2},
$$

are the transmission and reflection coefficients in the absence of $\mathrm{CP}$-violation [22, and

$$
\delta_{1}^{\text {refl }}=-\left.\delta_{1}^{\text {inc }}\right|_{\beta_{s} \rightarrow-\beta_{s}}, \quad \delta_{2}^{\text {refl }}=-\left.\delta_{2}^{\text {inc }}\right|_{\beta_{s} \rightarrow-\beta_{s}} .
$$

The unitary condition, $R_{s, B}^{(0)}+T_{s, B}^{(0)}=1$, holds as it should be. We find the expression for $c_{-}^{(+)}$by requiring the unitary condition for the reflection and transmission coefficients $R_{s, B}$ and $T_{s, B}$. Imposing that

$$
R_{s, B}+T_{s, B}=1
$$

we get

$$
c_{-}^{(+)}=-\frac{1}{\xi} \frac{\operatorname{Re}\left[I_{1}^{(s)}\right]}{\operatorname{Re}\left[I_{2}^{(s)} /\left(\xi-\alpha_{s}\right)\right]} .
$$

Now, we calculate the axial currents $\left(j_{A, s}^{3}\right)^{\text {inc }}$ and $\left(j_{A, s}^{3}\right)^{\text {refl }}$, analogously to the vectorial current. We get the following expressions:

$$
\begin{aligned}
\left(j_{A, s}^{3}\right)^{\text {inc }} & =(-1)^{s+1}\left|A_{s}^{(+)}\right|^{2}\left|\gamma_{+}\left(\alpha_{s}, \beta_{s}\right)\right|^{2} \\
& \times\left[\epsilon^{2}\left(1+\delta_{1}^{\text {inc }}\right)+\left|\beta_{s}\right|^{2}\left(1+\delta_{1}^{\text {inc }}+2 \delta_{2}^{\text {inc }}\right)\right], \\
\left(j_{A, s}^{3}\right)^{\text {refl }} & =\left.\left(j_{A, s}^{3}\right)^{\text {inc }}\right|_{\beta_{s} \rightarrow-\beta_{s}},
\end{aligned}
$$

where, here and in the following (see Eqs. (2.58), (2.63) and (2.64) ), we set $g_{-}=0$.

Finally, we are able to calculate the relevant quantity for the generation of a cosmological baryon asymmetry,

$$
\Delta R^{(s)}=R_{R \rightarrow L}^{(s)}-R_{L \rightarrow R}^{(s)},
$$

where

$$
\begin{aligned}
& R_{R \rightarrow L}^{(s)}=-\frac{\left(j_{L, s}^{3}\right)^{\text {refl }}}{\left(j_{R, s}^{3}\right)^{\text {inc }}}=\frac{\left(j_{A, s}^{3}\right)^{\text {refl }}-\left(j_{V, s}^{3}\right)^{\text {refl }}}{\left(j_{V, s}^{3}\right)^{\text {inc }}+\left(j_{A, s}^{3}\right)^{\text {inc }}}, \\
& R_{L \rightarrow R}^{(s)}=-\frac{\left(j_{R, s}^{3}\right)^{\text {refl }}}{\left(j_{L, s}^{3}\right)^{\text {inc }}}=\frac{\left(j_{A, s}^{3}\right)^{\text {refl }}+\left(j_{V, s}^{3}\right)^{\text {refl }}}{\left(j_{A, s}^{3}\right)^{\text {inc }}-\left(j_{V, s}^{3}\right)^{\text {inc }}} .
\end{aligned}
$$


Taking into account the expressions for the currents, we obtain

$$
\Delta R^{(s)}=2 R_{s, B}^{(0)}\left(\delta_{s, B}^{\mathrm{inc}}+\delta_{s, B}^{\mathrm{refl}}\right),
$$

where we have defined the quantities

$$
\delta_{s, B}^{\mathrm{inc}}=\frac{\xi}{2\left|\alpha_{s}\right|}\left[\frac{\gamma_{-}\left(-\alpha_{s}, \beta_{s}\right)}{\gamma_{+}\left(\alpha_{s}, \beta_{s}\right)} I_{2}^{(s)}+\text { c.c. }\right], \quad \delta_{s, B}^{\mathrm{refl}}=\left.\delta_{s, B}^{\mathrm{inc}}\right|_{\beta_{s} \rightarrow-\beta_{s}} .
$$

In the case of vanishing magnetic field, $\Delta R^{(s)}$ reduces to the same expression calculated in Ref. [21].

In order to study $\Delta R^{(s)}$ we have to know $f(x)$ and $g(x)$. As regards $f(x)$ we take the usual bubble wall profile

$$
f(x)=\frac{1+\tanh x}{2} .
$$

With the profile (2.58), the solutions $\phi_{ \pm}^{\left( \pm \alpha_{s}\right)}(x)$ of the unperturbed equation (2.23) may be written as

$$
\begin{aligned}
& \phi_{ \pm}^{\left(+\alpha_{s}\right)}(y)=y^{-\frac{\alpha_{s}}{2}}(1-y)^{\frac{\beta_{s}}{2}}{ }_{2} F_{1}\left[\frac{-\alpha_{s}+\beta_{s} \mp \xi}{2}+1, \frac{-\alpha_{s}+\beta_{s} \pm \xi}{2}, 1-\alpha_{s} ; y\right], \\
& \phi_{ \pm}^{\left(-\alpha_{s}\right)}(y)=y^{\frac{\alpha_{s}}{2}}(1-y)^{\frac{\beta_{s}}{2}}{ }_{2} F_{1}\left[\frac{\alpha_{s}+\beta_{s} \mp \xi}{2}+1, \frac{\alpha_{s}+\beta_{s} \pm \xi}{2}, 1+\alpha_{s} ; y\right]
\end{aligned}
$$

where $y=1-f(x)$, and ${ }_{2} F_{1}(a, b, c ; x)$ is the the hypergeometric function. Now, $\gamma_{ \pm}\left(\alpha_{s}, \beta_{s}\right)$ and $R_{s, B}^{(0)}$ are explicitly given by

$$
\gamma_{ \pm}\left(\alpha_{s}, \beta_{s}\right)=\frac{\Gamma\left(-\alpha_{s}+1\right) \Gamma\left(-\beta_{s}\right)}{\Gamma\left[\left(-\alpha_{s}-\beta_{s} \pm \xi\right) / 2\right] \Gamma\left[\left(-\alpha_{s}-\beta_{s} \mp \xi\right) / 2+1\right]},
$$

and

$$
R_{s, B}^{(0)}=\frac{\sin \left[(\pi / 2)\left(\alpha_{s}-\beta_{s}+\xi\right)\right] \sin \left[(\pi / 2)\left(\alpha_{s}-\beta_{s}-\xi\right)\right]}{\sin \left[(\pi / 2)\left(\alpha_{s}+\beta_{s}+\xi\right)\right] \sin \left[(\pi / 2)\left(\alpha_{s}+\beta_{s}-\xi\right)\right]} .
$$

The functional form of $g(x)$ is unknown. Following Ref. [21], we consider two expressions for $g(x)$ :

$$
\begin{aligned}
& g(x)=\Delta \theta f^{2}(x), \\
& g(x)=\Delta \theta f^{\prime}(x),
\end{aligned}
$$

where the parameter $\Delta \theta$ characterizes the magnitude of CP-violation.

In Figs. 1-4 we plot $\Delta R^{(s)} / \Delta \theta$ as a function of normalized energy $\epsilon^{*}=\epsilon a^{*}=E / m_{0}$, at fixed thickness $a^{*}=a / m_{0}=1 / \xi\left(a^{*}=5\right.$ in Figs. 1 and 3, $a^{*}=1$ in Fig. 2 and 4) for different values of the magnetic field $b^{*}=b a^{* 2}=e B / m_{0}^{2}$. We take $s=1$ (left panels) and $s=2$ (right panels), with $g(x)=\Delta \theta f^{2}(x)$ in Figs. 1,2, and $g(x)=\Delta \theta f^{\prime}(x)$ in Figs. 3,4. We found that $\Delta R^{(s)}$ display the following peculiar properties:

i) At fixed thickness of the wall (proportional to $1 / a^{*}$ ) and magnetic field, the absolute value of $\Delta R^{(s)}$ goes to zero when the energy of the incident particles approaches to infinity;

ii) The maximum value of $\left|\Delta R^{(s)}\right|$ vary both with the thickness of the wall and with the functional form of $g(x)$. In particular, we found that at fixed energy and magnetic field, $\left|\Delta R^{(s)}\right|$ is an increasing function of the thickness of the wall; 
iii) The presence of a magnetic field generates a reflection asymmetry between spin-up and spin-down particles. In particular, the effect of the magnetic field is to shift the values of $\Delta R^{(s)}$ (with respect to the case $B=0$ ) towards lower energies in the case $s=1$, and higher energies in the case $s=2$.

The peculiar global quantity in non-local defect mediated electroweak baryogenesis is the flux of lepton number radiated by the bubble wall. In the thermal frame of reference, it is given by (see e.g. Ref. [16]):

$$
\Phi_{L}=\int \frac{d^{3} k}{(2 \pi)^{3}} e^{-k / T} L(\mathbf{k}) \cos \vartheta_{R}
$$

where $T$ is the temperature and, considering only one type of particle, $L(\mathbf{k})=l|R(\mathbf{k})|^{2}$. Here, $R(\mathbf{k})$ is the reflection amplitude for a particle of momentum $\mathbf{k}, \vartheta_{R}$ is the angle of reflection off the advancing wall, $k=|\mathbf{k}|$, and $l$ is the lepton number. In the case of an infinitely planar wall only the motion of fermions perpendicular to the wall is important, and then we have $\Phi_{L}(B) \propto \int d E e^{-E / T} \Delta R(E, B)$, where $E$ is the energy of the scattered particle, and $\Delta R=R_{R \rightarrow L}-R_{L \rightarrow R}$. Indeed, the relevant quantity for the generation of a cosmological baryon asymmetry turns out to be $\Phi_{L}^{\text {tot }}(B)+\Phi_{L}^{\text {tot }}(-B)$, where

$$
\Phi_{L}^{t o t}(B)=\sum_{s=1,2} \Phi_{L}^{(s)}(B)=\alpha \sum_{s=1,2} \int d E e^{-E / T} \Delta R^{(s)}(E, B) .
$$

Here, $\alpha>0$ is a numerical factor, and $s$ refers to the spin of the scattered particle. It is straightforward to verify that the following transformation law hold:

$$
\Delta R^{(s)}(-B)=\Delta R^{(\bar{s})}(B),
$$

where $\bar{s}=1$ if $s=2$, and $\bar{s}=2$ if $s=1$. Finally, taking into account the previous equations, we have

$$
\Phi_{L}^{(s)}(B)+\Phi_{L}^{(s)}(-B)=\Phi_{L}^{(s)}(B)+\Phi_{L}^{(\bar{s})}(B)=\Phi_{L}^{t o t}(B),
$$

and

$$
\Phi_{L}^{t o t}(B)+\Phi_{L}^{t o t}(-B)=2 \Phi_{L}^{\text {tot }}(B) .
$$

In general, the quantity $\Phi_{L}^{\text {tot }}(B)$ is not null. For example, from Fig. 1, we get that $\Phi_{L}^{(1)}(B)$ and $\Phi_{L}^{(2)}(B)$ are strictly negative quantities. Hence, in this case we have $\Phi_{L}^{\text {tot }}(B)=$ $\Phi_{L}^{(1)}(B)+\Phi_{L}^{(2)}(B)<0$, and then, in general, the total lepton number flux radiated by an infinitely planar wall is different than zero.

\section{Conclusions}

In this work we have investigate a baryogenesis scheme at a first order electroweak phase transition in which fermions interact with a CP-violating thick bubble wall in the background of an uniform magnetic field perpendicular to the wall.

We have studied the net flux by the difference $\Delta R^{(s)}$ of the reflection coefficients of the left-handed and right-handed fermions incident from the unbroken phase at the expanding bubble wall. We have found that the presence of a magnetic field generates a reflection asymmetry between spin-up and spin-down particles. Moreover, we have showed that the 
only effect of the magnetic field is to shift the values of $\Delta R^{(s)}$ (with respect to the case of vanishing magnetic field) towards lower energies in the case of spin-up particles, and higher energies in the case of spin-down particles.

In non-local defect mediated electroweak baryogenesis, the total baryon asymmetry turns out to be proportional to the total lepton number flux radiated by a bubble wall, $\Phi_{L}^{t o t}(B)-\Phi_{L}^{t o t}(-B)$. We have showed that, in general, this quantity is not null. In any case, the cosmological implications of a non-vanishing lepton number flux are beyond the aim of the present paper. We deserve such an analysis to a future work.

\section{Acknowledgments}

We would like to thank the referee for his/her insightful comments about the importance,

in non-local defect mediated electroweak baryogenesis, of the lepton number flux radiated by a bubble wall. 


\section{Appendix}

\section{A Transmitted wave function}

In this Appendix we calculate the transmitted wave function. In the same way it is straightforward to calculate the incident and reflected wave functions.

The wave function to the first order is given by Eq. (2.16), that we rewrite for simplicity:

$$
\Psi(\tau, x) \simeq\left[\left(\sigma \epsilon \gamma^{0}+i \gamma^{3} \frac{d}{d x}+\xi f+b x \gamma^{2}\right)\left(\psi^{(0)}+\psi^{(1)}\right)+i \xi g \gamma_{5} \psi^{(0)}\right] e^{-i \sigma \epsilon \tau} .
$$

Hence, in order to calculate the asymptotic form of $\Psi(\tau, x)$, it is necessary to know the asymptotic form of $\psi^{(0)}(x)$ and $\psi^{(1)}(x)$. Taking into account Eqs. (2.24) and (2.28) we obtain, for positive energy solutions and in the limit $x \rightarrow+\infty$,

$$
\left[\psi^{(0)}(x)\right]_{\sigma=+1}^{+\infty}=A_{s}^{(+)} u_{+}^{s} e^{\alpha_{s} x} .
$$

As regards $\psi^{(1)}(x)$ we start with Eq. (2.33). Taking into account Eqs. (2.24), (2.25), and Eqs. (2.34), (2.35) we have

$$
\left[\psi_{s}^{(1)}(x)\right]_{\sigma=+1}^{+\infty}=A_{s}^{(+)} \frac{(-1)^{s} \xi}{2 \alpha_{s}}\left[I_{1}^{(s)}+c_{-}^{(+)} I_{2}^{(s)}\right] u_{-}^{s} e^{\alpha_{s} x}
$$

Remembering that $\lim _{x \rightarrow+\infty} f(x)=1$, the transmitted wave function turns out to be

$$
\begin{aligned}
{[\Psi(\tau, x)]_{\sigma=+1}^{\operatorname{tran}} } & =\left[\left(\epsilon \gamma^{0}+i \gamma^{3} \frac{d}{d x}+\xi+b x \gamma^{2}\right)\left(\left[\psi_{s}^{(0)}(x)\right]_{\sigma=+1}^{+\infty}+\left[\psi_{s}^{(1)}(x)\right]_{\sigma=+1}^{+\infty}\right)\right. \\
& \left.+i \xi g_{+} \gamma_{5}\left[\psi_{s}^{(0)}(x)\right]_{\sigma=+1}^{+\infty}\right] e^{-i \epsilon \tau} .
\end{aligned}
$$

Finally, inserting Eqs. (A.2) and (A.3) into Eq. (A.4), we get

$$
\begin{aligned}
{\left[\Psi_{s}(\tau, x)\right]_{\sigma=+1}^{\operatorname{tran}} } & =A_{s}^{(+)}\left\{\left[\xi-\alpha_{s}+\frac{(-1)^{s} \xi \epsilon}{2 \alpha_{s}}\left(I_{1}^{(s)}+c_{-}^{(+)} I_{2}^{(s)}\right)\right] u_{+}^{s}\right. \\
& \left.+\left[\epsilon+\frac{(-1)^{s}}{2 \alpha_{s}} \xi\left(\xi+\alpha_{s}\right)\left(I_{1}^{(s)}+c_{-}^{(+)} I_{2}^{(s)}\right)-(-1)^{s} \xi g_{+}\right] u_{-}^{s}\right\} \\
& \times e^{-i \epsilon \tau+\alpha_{s} x} .
\end{aligned}
$$

Following the same arguments of Appendix in Ref. [21, it is possible to redefine $A_{s}^{(+)}$as

$$
A_{s}^{(+)}\left[1+(-1)^{s} \frac{\xi\left(\xi+\alpha_{s}\right)}{2 \epsilon \alpha_{s}} c_{-}^{(+)} I_{2}^{(s)}\right]\left[1-(-1)^{s} \frac{\xi}{2 \epsilon} g_{-}\right] \rightarrow A_{s}^{(+)} \text {. }
$$

Taking into account Eq. (A.6), it is straightforward to cast Eq. (A.5) into the form of Eq. (2.36). 


\section{References}

[1] A.D. Sakharov, Pis'ma Zh. Eksp. Teor. Fiz. 5, 32 (1967) [JETP Lett. 5, 24 (1967)].

[2] G.'t Hooft, Phys. Rev. Lett. 37, 8 (1976); Phys. Rev. D14, 3432 (1976).

[3] F. Kleinkhamer and M. Manton, Phys. Rev. D30, 2212 (1984).

[4] V.A. Kuzmin, V.A. Rubakov and M.E. Shaposhnikov, Phys. Lett. B155, 36 (1985).

[5] B. Aubert et al. [BABAR Collaboration], hep-ex/0207070 K. Abe et al. [Belle Collaboration], hep-ex/0207098.

[6] C.L. Bennet et al., Astroph. J. Suppl. 148, 1 (2003).

[7] M.E. Shaposnikov, Phys. Lett. B277, 324 (1992) [Erratum-ibid. B282, 483 (1992)].

[8] G.R. Farrar and M.E. Shaposhnikov, Phys. Rev. D50, 774 (1994).

[9] S.M. Barr, G. Segre, and H.A. Weldon, Phys. Rev. D20, 2492 (1979).

[10] M.E. Carrington, Phys. Rev. D45, 2933 (1992).

[11] K. Kajantie, M. Laine, K. Rummukainen, and M.E. Shaposhnikov, Nucl. Phys. B466, 189 (1996).

[12] M.E. Shaposhnikov, Nucl. Phys. JETP Lett. 44, 465 (1986); Nucl. Phys. B287, 757 (1987); Nucl. Phys. B299, 797 (1988).

[13] K. Hagiwara et al. [Particle data Group Collaboration] Phys. Rev. D66, 01001 (2002).

[14] J.S. Langer, Annals Phys. 54, 258 (1969).

[15] A.D. Linde, Phys. Lett. B100, 37 (1981).

[16] A. Cohen, D. Kaplan, and A. Nelson, Phys. Lett. B245, 561 (1990); Nucl. Phys. B349, 727 (1991); Nucl. Phys. B373, 453 (1992); M. Joyce, T. Prokopec, and N. Turok, Phys. Lett. B338, 269 (1994); Phys. Rev. Lett. 75, 1695 (1995).

[17] L.D. McLerran, M.E. Shaposhnikov, N. Turok, and M.B. Voloshin, Phys. Lett. B256, 451 (1991).

[18] N. Turok and J. Zadrozny, Nucl. Phys. B358, 471 (1991).

[19] M. Joyce, T. Prokopec, and N. Turok, Phys. Lett. B339, 312 (1994); Phys. Rev. D53, 2930 (1996).

[20] A. Ayala, J. Jalilian-Marian, L. McLerran, and A.P. Visher, Phys. Rev. D49, 5559 (1994).

[21] K. Funakubo, A. Kakuto, S. Otsuki, K. Takenaga, and F. Toyoda, Phys. Rev. D50, 1105 (1994); hep-ph/9405422. 
[22] P. Cea, G.L. Fogli, and L. Tedesco, Mod. Phys. Lett. A15, 1755 (2000); L. Campanelli, P. Cea, G.L. Fogli, and L. Tedesco, Phys. Rev. D65, 085004 (2002).

[23] A.D. Dolgov, astro-ph/0306443; D. Grasso and H.R. Rubinstein, Phys. Rept. 348, 163 (2001); L.M. Widrow, Rev. Mod. Phys. 74, 775 (2003); For a recent review on large-scale magnetic fields in the Universe see: M. Giovannini, Int. J. Mod. Phys. D13, 391 (2004).

[24] C.H. Hogan, Phys. Rev. Lett. 51, 1488 (1983).

[25] T. Vachaspati, Phys. Lett. B265, 258 (1991); G. Baym, D. Bodeker, and L. McLerran, Phys. Rev. D53, 662 (1996).

[26] A. Nelson, D. Kaplan, and A. Cohen, Nucl. Phys. B373, 453 (1992).

[27] R. Courant and D. Hilbert, Methods of Mathematical Physics (Interscience, New York, 1953). 

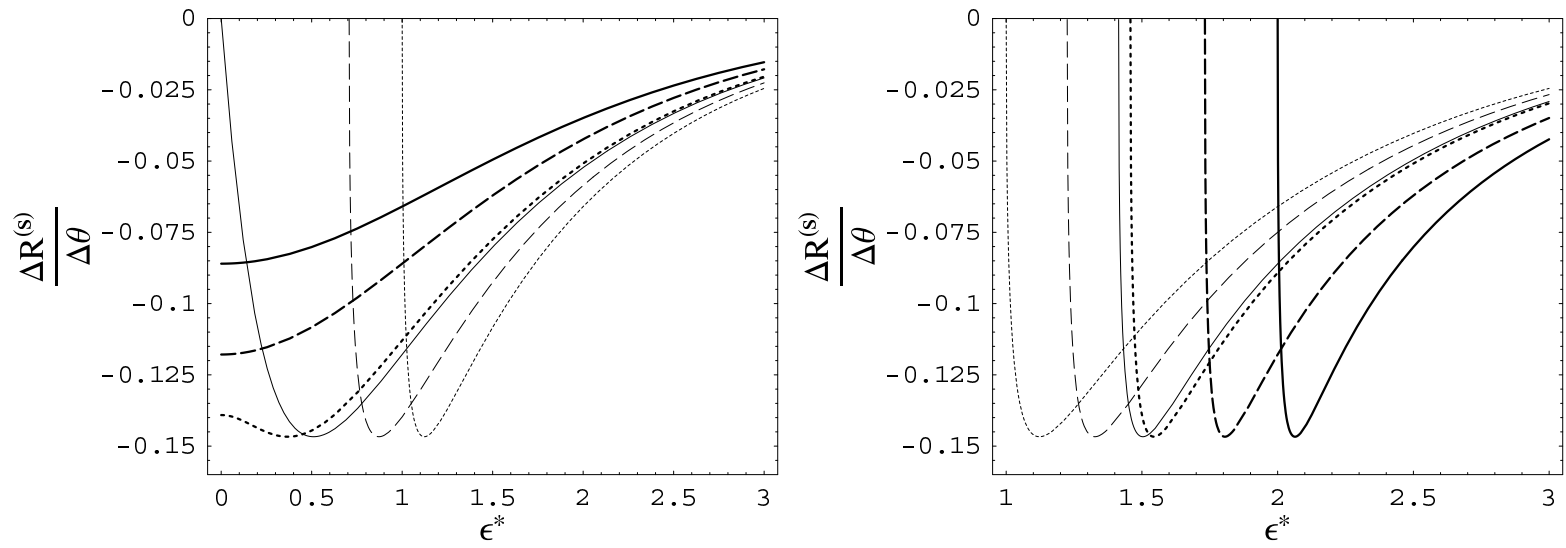

Figure 1: $\Delta R^{(s)} / \Delta \theta$ versus $\epsilon^{*}$, with $s=1$ (left panel) and $s=2$ (right panel), for different values of normalized magnetic field $b^{*}$, in the case $g=\Delta \theta f^{2}$ and $a^{*}=5$. Thick solid line: $b^{*}=3$; thick long dashed line: $b^{*}=2$; thick short dashed line: $b^{*}=1.125$; thin solid line: $b^{*}=1$; thin long dashed line: $b^{*}=0.5$; thin short dashed line: $b^{*}=0$.
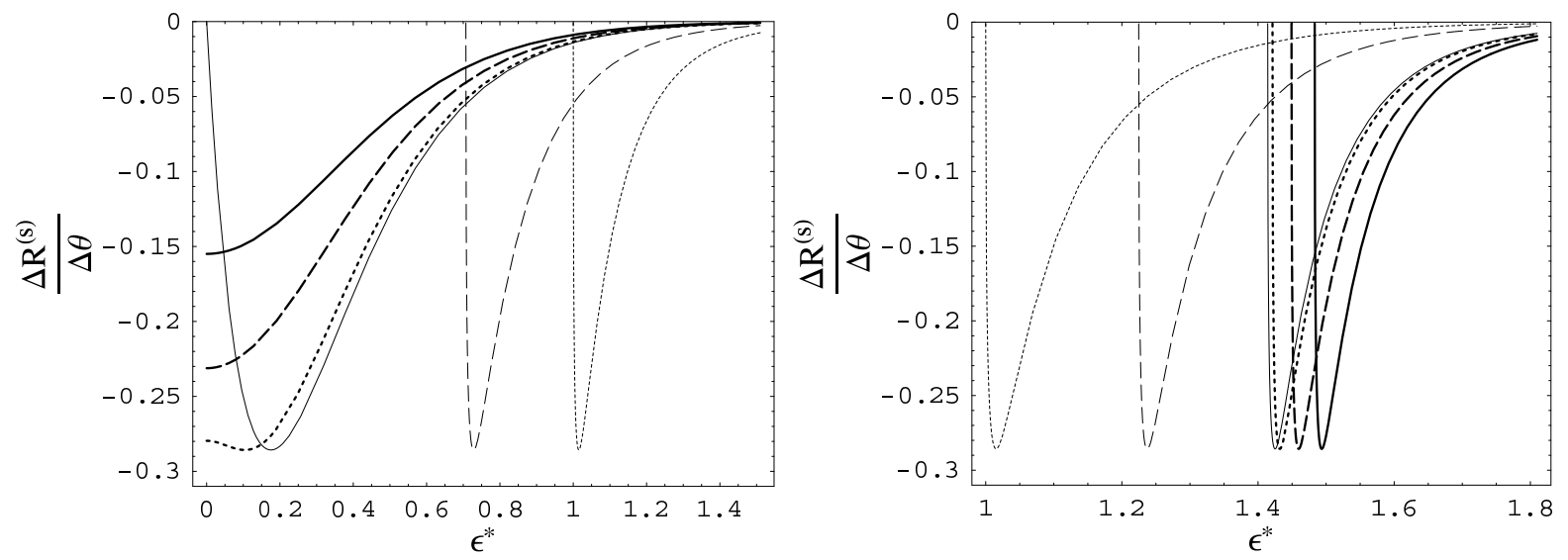

Figure 2: $\Delta R^{(s)} / \Delta \theta$ versus $\epsilon^{*}$, with $s=1$ (left panel) and $s=2$ (right panel), for different values of normalized magnetic field $b^{*}$, in the case $g=\Delta \theta f^{2}$ and $a^{*}=1$. Thick solid line: $b^{*}=1.2$; thick long dashed line: $b^{*}=1.1$; thick short dashed line: $b^{*}=1.02$; thin solid line: $b^{*}=1$; thin long dashed line: $b^{*}=0.5$; thin short dashed line: $b^{*}=0$. 

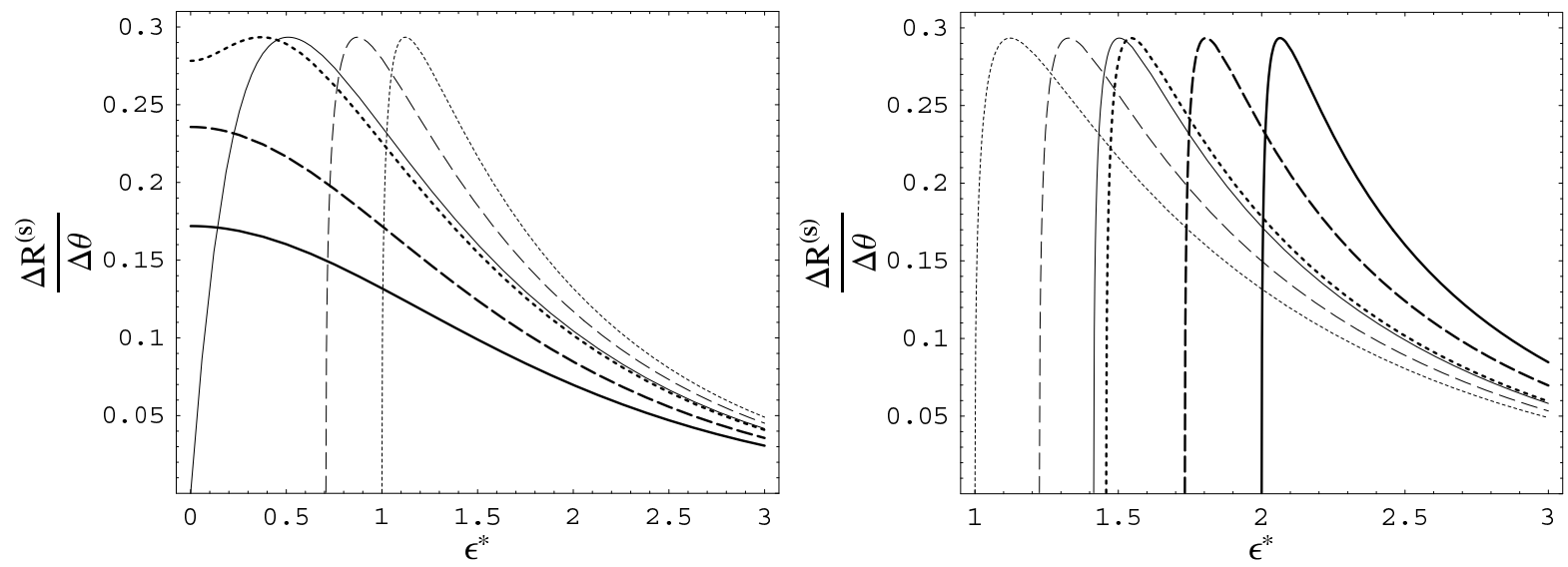

Figure 3: $\Delta R^{(s)} / \Delta \theta$ versus $\epsilon^{*}$, with $s=1$ (left panel) and $s=2$ (right panel), in the case $g=\Delta \theta f^{\prime}$ and $a^{*}=5$. The values of $b^{*}$ are the same as in Fig. 1 .
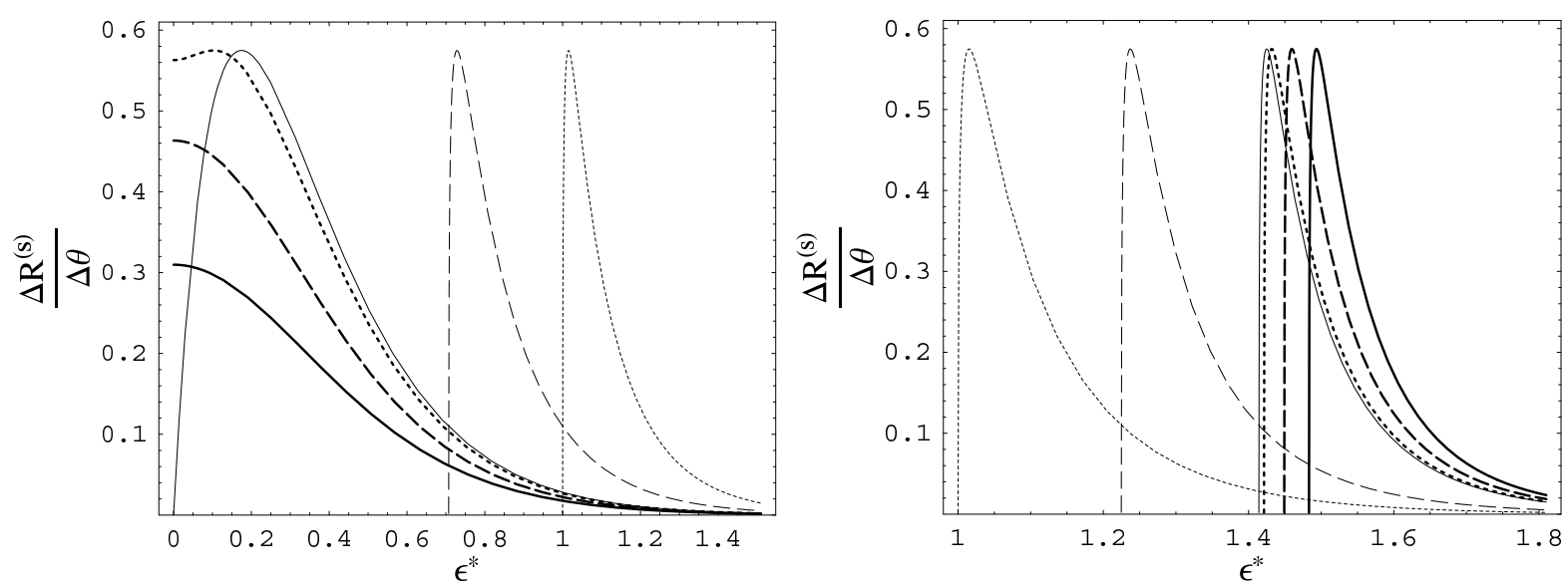

Figure 4: $\Delta R^{(s)} / \Delta \theta$ versus $\epsilon^{*}$, with $s=1$ (left panel) and $s=2$ (right panel), in the case $g=\Delta \theta f^{\prime}$ and $a^{*}=1$. The values of $b^{*}$ are the same as in Fig. 2. 